\title{
Répartition des nutriments dans un sol brun acide développé sur tuf (Beaujolais, France). Conséquences pour l'évolution de la fertilité minérale à long terme
}

\author{
A Ezzaïm*, MP Turpault, J Ranger \\ Équipe cycles biogéochimiques, centre Inra de Nancy, 54280 Champenoux, France
}

(Reçu le 24 avril 1996 ; accepté le 24 juillet 1996)

\begin{abstract}
Summary - Nutrient distribution in an acid brown soil developed from a volcanic tuff bedrock (Beaujolais, France). Consequences for the long-term fertility. The study of soil fertility, often measured only by its short-term component (ie, exchangeable cation reserves), appears insufficient and can mask different situations. This work shows that the middle and long-term fertility characterized by the nutrient content of minerals of three acid brown soils was very different although their exchangeable cation reserves were comparable. The middle and long-term fertility of these soils corresponds to reserves of nutrient elements in the fresh tuff, in the weathered tuff and in the coarse soil fractions originating from the desquamation of the weathered tuff. Differences in the fertility of these three soils were due to the colluviation and to the intense hydrothermalism that affected the very top layer of tuff and provoked a major calcium depletion in the bedrock. Calcium appeared to be the first limiting factor for plant nutrition in this ecosystem. Only the flux of calcium originating from the dissolution of calcic primary minerals in the soil fractions $(>2 \mu \mathrm{m})$ was able to maintain the temporary reserve of this element on the adsorbing complex and to meet the nutritional demands of the Douglas-fir stand. The stability of this ecosystem therefore depends mainly on the rate of calcium flux originating from minerals located in the coarse soil fractions, assuming that the organic matter compartment is stable.
\end{abstract}

mineral fertility / long-term reserve / acid brown soil / ecosystem stability / Douglas (Pseudotsuga menziessii Franco)

Résumé - L'étude de la fertilité d'un sol, souvent mesurée uniquement par sa composante à court terme (stock des éléments échangeables), apparaît insuffisante et peut dissimuler des situations très différentes. Ce travail montre que la fertilité à moyen et à long terme (éléments nutritifs dans les minéraux du sol) de trois sols bruns acides du massif forestier des Aiguillettes est très différente, bien que leurs stocks en éléments nutritifs échangeables soient comparables. La fertilité à moyen et à long terme de ces sols correspond au stock d'éléments nutritifs du tuf sain, de la pellicule d'altération et des fractions granulométriques issues de cette pellicule par desquamation. La différence entre la fertilité

\footnotetext{
* Correspondance et tirés à part
}

Tél : (33) 0323394175 ; fax : (33) 0383394175 
des trois profils étudiés est due au colluvionnement et à l'hydrothermalisme intense ayant affecté le toit du tuf, et conduisant notamment à un appauvrissement important en calcium. Cet élément apparaît comme le premier facteur limitant pour la nutrition des peuplements dans cet écosystème. Seul le flux de calcium issu de la dissolution des minéraux primaires calciques des fractions du sol supérieures à $2 \mu \mathrm{m}$ est capable de maintenir le stock temporaire de cet élément sur le complexe adsorbant et de subvenir aux exigences nutritives du peuplement de douglas : la stabilité de cet écosystème dépend donc en grande partie de l'importance de ce flux dans l'hypothèse de stabilité du compartiment organique.

fertilité minérale / stock à long terme / sol brun acide / stabilité de l'écosystème / douglas = Pseudotsuga menziessii Franco

\section{INTRODUCTION}

Le pool de cations basiques adsorbés sous forme échangeable sur les colloïdes organiques et minéraux représente la fertilité chimique actuelle et à court terme d'un sol forestier. Les éléments contenus dans les minéraux du sol (hérités de la roche mère ou minéraux secondaires) caractérisent la fertilité à moyen et à long terme. Ces éléments vont être libérés lentement dans les solutions du sol au cours de simples réactions d'échange ionique ou de réactions d'altération physicochimique (fragmentation, hydrolyse, acidolyse, complexolyse), souvent d'origine biologique pour ce qui concerne l'acidolyse et la complexolyse (Bonneau, 1995). La fraction supérieure à $2 \mu \mathrm{m}$ contenant les minéraux du sol représente donc la fertilité à moyen et à long terme. La localisation des éléments nutritifs au sein des différentes classes granulométriques de cette fraction grossière varie en fonction de leur composition minéralogique et chimique. Le flux d'altération qui en résulte sera d'autant plus élevé que les surfaces spécifiques des minéraux exposés au contact des solutions seront importantes (Stanley et Barber, 1984).

Cinq types de réactions sont mises en jeu lors de l'altération supergène des minéraux du sol : microdivision, dissolution, transformation, néoformation et échange d'ions. L'altération des minéraux par voies de dissolution, de transformation et de néoformation contribue directement au flux d'altération et conduit à la formation des minéraux secondaires ou à la libération d'ions en solution.

Le flux d'altération détermine, en matière de bilan, une grande partie de la résilience des écosystèmes traités extensivement. Leur stabilité se traduit par des flux entrées-sorties équilibrés du cycle biogéochimique (Ranger et al, 1995a). Les apports atmosphériques (pluies et dépôts secs) et le flux d'altération représentent les entrées du système ; le drainage et les exportations par les récoltes représentent les sorties.

Depuis 1993, le massif forestier des Aiguillettes (Beaujolais, France) a fait l'objet d'un suivi hydrochimique et de recherches sur le fonctionnement du cycle biogéochimique des éléments majeurs, au cours des principaux stades de développement d'un peuplement forestier de douglas. Dans cet écosystème, les apports atmosphériques, l'immobilisation dans le peuplement et les pertes par drainage ont été quantifiés (Ranger et al, 1995b ; Marques, 1996). Cependant, ce suivi ne prend pas en compte le flux d'éléments issu de l'altération des minéraux du sol et le bilan entrées-sorties ne permet pas de le calculer; seul le flux d'altération correspondant à la stabilité de l'écosystème peut être déduit. Pour évaluer ce dernier, une méthodologie originale est adoptée. Elle consiste, après avoir quantifié les minéraux en fonction de la granulométrie, à estimer ce flux à l'aide du modèle géochimique Profile (Sverdrup et Warfvinge, 1988) qui prend en compte les caractéristiques minéralogiques et physicochimiques des différents 
horizons, la qualité des solutions et le prélèvement par la végétation. Le flux d'altération dépend des conditions physiques du milieu, de la surface spécifique des minéraux, de la composition des minéraux, de la composition chimique et du temps de résidence de la solution à leur contact. La première étape de ce travail consiste à caractériser la phase solide en fonction de la granulométrie.

L'objectif de cet article est de quantifier les stocks de nutriments (Ca, K, Mg et P) et de les localiser précisément dans les différentes classes granulométriques des sols d'une toposéquence de trois profills de sol, dans le but de comparer cette réserve aux éléments nutritifs immobilisés dans la biomasse, aux apports atmosphériques totaux et au drainage à $120 \mathrm{~cm}$ de profondeur durant une révolution forestière du douglas. Ces résultats permettront d'apporter une première réponse sur la caractérisation de la fertilité du sol de cet écosystème et de son évolution possible.

\section{MATÉRIELS ET MÉTHODES}

\section{Matériels}

\section{Placettes forestières}

Deux placettes forestières principales, correspondant à deux peuplements de douglas âgés de 40 et 60 ans, ont été choisies dans le massif forestier des Aiguillettes sur la commune de Vauxrenard (bordure orientale du Massif central, nord-est de Beaujeu).

La roche mère la plus représentative dans ces placettes est volcanique, de type tuf d'âge Viséen (Carbonifêre). Le grès quartzofeldspathique daté du Trias coiffant la montagne des Aiguillettes colluvionne partiellement ces deux placettes. Une troisième placette constituée d'un jeune peuplement d'épicéas, située au contact géologique tuf/grès, est sélectionnée afin de mieux comprendre le phénomène de colluvionnement des placettes principales d'un point de vue chimique et granulométrique.

Ces trois placettes constituent une toposéquence à une altitude située entre 780 et $790 \mathrm{~m}$ avec des pentes faibles $\left(<10^{\circ}\right.$ ) (fig 1 ). Le climat actuel est caractérisé par des températures moyennes de $7{ }^{\circ} \mathrm{C}$ et une pluviosité annuelle de $1000 \mathrm{~mm}$.

\section{Caractéristiques des roches mères}

L'histoire géologique du massif forestier des Aiguillettes s'intègre à celle du faisceau de la Loire (Morel, 1976 ; Bertaux et Rubiello, 1981 ; Sider et Leistel, 1986), portion de la chaîne hercynienne dans le nordest du Massif central. Ce massif forestier

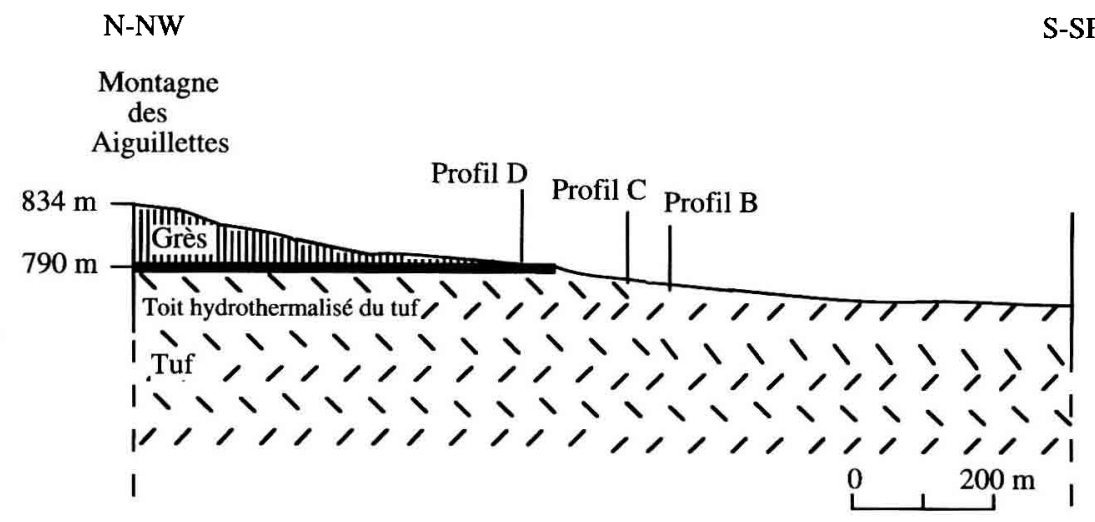

Fig 1. Coupe N-NW/S-SE du site d'étude. 
coiffé de grès quartziques à quartzo-feldspathique du Trias est constitué à la base de tuf d'âge viséen supérieur dont le "toit " est hydrothermalisé (fig 1). Ce tuf volcanique est formé d'un assemblage de verre recristallisé et de phénocristaux (quartz, andésine [37\% anorthite], albite, orthose, biotite, chlorite, micas blancs, épidote et apatite) (Ezzaïm et al, soumis pour publication). Il est riche en éléments nutritifs $(\mathrm{CaO}: 2 \% ; \mathrm{MgO}: 1,9 \%)$, contrairement aux grès $(\mathrm{CaO}: 0,2 \% ; \mathrm{MgO}: 0,03 \%$; tableau I). Le toit du tuf est affecté par une altération hydrothermale intense à micas blancs, quartz et hématite ; les phénocristaux et la mésostase du tuf sont partielle- ment ou totalement remplacés par ces minéraux en fonction du degré de l'hydrothermalisme.

L'altération supergène des cailloux de tuf sain non affecté par l'hydrothermalisme observé à la base de la montagne des Aiguillettes se traduit par le développement d'une pellicule d'altération de couleur jaunâtre tout autour du cœeur sain du tuf de couleur gris noir. Cette pellicule est fortement appauvrie en éléments nutritifs (analyse TAMI ; tableau I). Cependant, le tuf hydrothermalisé affecté par l'altération supergène devient très poreux et friable, conduisant à une désagrégation massive de la roche.

Tableau I. Composition chimique totale (\% masse) du tuf sain moyen (TSM), du tuf altéré moyen des profils B et C (TAM1) et du profil D (TAM2), du grès (GQ1 et GV6) et de la terre fïne (TF) du profil $\mathrm{B}$ à différentes profondeurs.

\begin{tabular}{|c|c|c|c|c|c|c|c|c|c|}
\hline & $T S M$ & $T A M I$ & $T A M 2$ & $G Q I$ & GV6 & $T F-5 \mathrm{~cm}$ & $T F-15 \mathrm{~cm}$ & $T F-37,5 \mathrm{~cm}$ & $T F-115 \mathrm{~cm}$ \\
\hline \multirow[t]{2}{*}{$\mathrm{SiO}_{2}$} & 66,9 & 67,8 & 69,8 & 92,7 & 93,9 & 66,7 & 69,0 & 72,0 & 65,1 \\
\hline & 0,62 & 1,09 & & & & & & & \\
\hline \multirow{2}{*}{$\mathrm{Al}_{2} \mathrm{O}_{3}$} & 15,0 & 14,2 & 14,3 & 2,7 & 2,8 & 11,8 & 12,9 & 13,4 & 17,6 \\
\hline & 0,45 & 1.47 & & & & & & & \\
\hline \multirow[t]{2}{*}{$\mathrm{MgO}$} & 1,9 & 1,8 & 1,5 & 0,0 & 0,0 & 0,8 & 0,9 & 0,9 & 1,5 \\
\hline & 0,09 & 0,30 & & & & & & & \\
\hline \multirow[t]{2}{*}{$\mathrm{CaO}$} & 2,0 & 0,6 & 0,2 & 0,2 & 0,2 & 0,1 & 0,1 & 0,1 & 0,2 \\
\hline & 0,41 & 0,40 & & & & & & & \\
\hline \multirow[t]{2}{*}{$\mathrm{Fe}_{2} \mathrm{O}_{3}$} & 3,7 & 3,9 & 3,0 & 0,1 & 0,1 & 3,0 & 3,1 & 3,2 & 4,2 \\
\hline & 0.15 & 0,52 & & & & & & & \\
\hline \multirow[t]{2}{*}{$\mathrm{TiO}_{2}$} & 0,6 & 0,7 & 0,7 & 0,1 & 0,1 & 0,6 & 0,7 & 0,7 & 0,6 \\
\hline & 0,01 & 0.05 & & & & & & & \\
\hline \multirow[t]{2}{*}{$\mathrm{P}_{2} \mathrm{O}_{5}$} & 0,2 & 0,2 & 0,2 & 0,1 & 0,1 & 0,1 & 0,1 & 0,1 & 0,1 \\
\hline & 0,03 & 0,07 & & & & & & & \\
\hline \multirow[t]{2}{*}{$\mathrm{Na}_{2} \mathrm{O}$} & 2,9 & 1,4 & 0,1 & 0,1 & 0,1 & 0,5 & 0,5 & 0,4 & 0,8 \\
\hline & 0,36 & 0,45 & & & & & & & \\
\hline \multirow[t]{2}{*}{$\mathrm{K}_{2} \mathrm{O}$} & 4,1 & 4,9 & 5,0 & 2,1 & 1,7 & 3,7 & 3,9 & 4,1 & 4,9 \\
\hline & 0.47 & 0,61 & & & & & & & \\
\hline \multirow[t]{2}{*}{ Paf } & 1,2 & 2,6 & 3,7 & 0,5 & 0,7 & 11,9 & 7,8 & 4,9 & 4,6 \\
\hline & 0,30 & 0,76 & & & & & & & \\
\hline $\mathrm{Da}$ & 2,70 & 2,22 & 2,51 & 2,50 & & & & & \\
\hline
\end{tabular}

Chiffres en italique : écarts types; $\mathrm{Fe}_{2} \mathrm{O}_{3}$ : calculé à partir du fer total ; Paf : perte au feu à $1000^{\circ} \mathrm{C}$; Da : densité apparente. 


\section{Profils d'altération}

Trois profils pédologiques ont été creusés dans les trois placettes forestières choisies et constituent une toposéquence. Deux profils ( $B$ et $C$ ) sont situés dans les deux placettes forestières de douglas $(40,60$ ans) et un troisième profil $\mathrm{D}$ situé dans une jeune plantation d'épicéas, au nord des profils $\mathrm{B}$ et $\mathrm{C}$ (fig 1).

Les sols sont de type brun acide et classés comme alocrisols typiques suivant le Référentiel pédologique (1992). L'humus est de type moder pour les profils B et C. Pour le profil D, l'horizon humifère superficiel a été noté lors de la plantation du jeune peuplement. Les caractéristiques physicochimiques des sols sont récapitulées dans le tableau II.

Les analyses chimiques totales de la terre fine brute (TF $;<2 \mathrm{~mm}$ ) montrent qu'elle s'est fortement appauvrie en certains éléments nutritifs par rapport à la roche mère ; il ne reste que des traces de calcium $(\mathrm{CaO}$ : 0,1 à $0,2 \%$ pour les profils $B$ et $C$ et 0,07 à $0,03 \%$ pour le profil D).

\section{Méthodes}

La mesure de la densité du sol et la quantification de ses différentes classes granulométriques sont effectuées à partir de trois fosses creusées à proximité des profils $\mathrm{B}$, $\mathrm{C}$ et $\mathrm{D}$, dont les dimensions sont de $2 \mathrm{~m}$ de longueur, $1 \mathrm{~m}$ de largeur et $1,20 \mathrm{~m}$ de profondeur. Quatre horizons $(0-10 \mathrm{~cm}, 10-30$ $\mathrm{cm}, 30-60 \mathrm{~cm}, 60-120 \mathrm{~cm}$ ) ont été individualisés pour les profils $\mathrm{B}$ et $\mathrm{C}$ et trois horizons $(0-30 \mathrm{~cm}, 30-65 \mathrm{~cm}, 65-115 \mathrm{~cm})$ pour le profil D. L'épaisseur des trois profils n'est pas systématique car la différenciation des horizons minéraux et organiques n'est pas nette au niveau du profil D.

La totalité du sol de chaque couche est tamisée à l'aide d'un tamis à maille carrée $(4 \times 4 \mathrm{~cm})$. Le refus et la fraction résiduelle
$(<4 \mathrm{~cm})$ sont pesés à l'état humide et un échantillon représentatif de $5 \mathrm{~kg}$ est prélevé. À partir de cet échantillon séché à $20^{\circ} \mathrm{C}$, différentes classes granulométriques sont séparées et quantifiées : inférieure à $2 \mathrm{~mm}$; $2-5 \mathrm{~mm} ; 5-10 \mathrm{~mm} ; 10-20 \mathrm{~mm} ; 20-40$ $\mathrm{mm}$. De plus, dans les classes granulométriques de la fraction supérieure à $2 \mathrm{~mm}$, les cailloux et les gravillons de tuf et de grès sont identifiés, triés et pesés.

À partir des densités apparentes du tuf sain et altéré et de l'épaisseur moyenne de la pellicule d'altération des cailloux et des gravillons, la masse de tuf sain et altéré est déterminée. Afin de simplifier la présentation des données, des classes granulométriques sont regroupées. Par conséquent, le sol est divisé en trois classes granulométriques principales et les résultats sont exprimés en masse par hectare rapportée à une siccité à $110^{\circ} \mathrm{C}: 1:$ la terre fine $(\mathrm{TF}:<2$ $\mathrm{mm}) ; 2$ : les gravillons (GR : $2-20 \mathrm{~mm})$; et 3 : les cailloux + blocs (CB : $>20 \mathrm{~mm}$ ). Connaissant la masse et la densité du tuf et du grès, la masse de la terre fine et le volume total de chaque couche, la densité de la terre fine est calculée pour chaque profil.

Après destruction de la matière organique de la terre fine par l'eau oxygénée à $30^{\circ} \mathrm{C}$, les fractions d'argiles et de limons fins sont séparćes en allonge et les fractions de limons grossiers, de sables fins et de sables grossiers sont séparées par tamisage (Robert et Tessier, 1974).

Les analyses chimiques totales sont réalisées sur la terre fine totale brute et sur les différentes fractions de la terre fine après destruction de la matière organique. Seuls les résultats concernant le potassium, le magnésium, le calcium et le phosphore sont présentés ici.

La mesure de la capacité d'échange cationique ( $T$ ) est effectuée sur les échantillons de terre fine selon la méthode décrite par Espiau et Peyronel (1976).

À partir des analyses précédemment décrites (granulométrie, analyses chimiques 


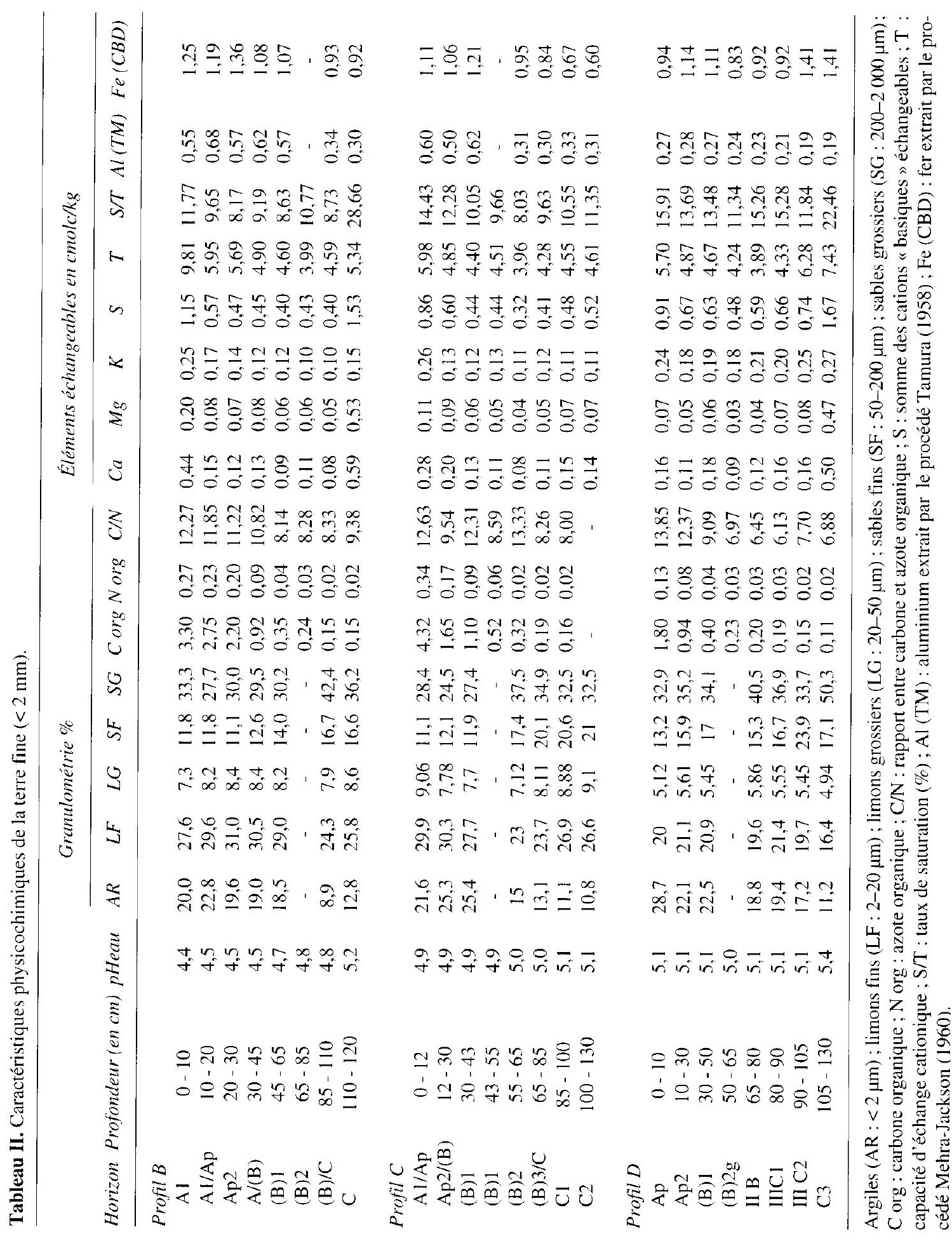


totales, capacité d'échange cationique de la terre fine et sa garniture cationique), la réserve totale d'éléments nutritifs dans les principales classes granulométriques du sol ainsi que le stock d'éléments échangeables dans la terre fine ont été quantifiés.

\section{RÉSULTATS}

\section{Granulométrie du sol (tableau III)}

Les sols sont moyennement caillouteux. La terre fine dans les profils $\mathrm{B}$ et $\mathrm{C}$ représente en moyenne $72 \%$ de la masse totale de l'horizon de surface $0-30 \mathrm{~cm}(2000 \mathrm{t} / \mathrm{ha})$, elle représente $65 \%$ de l'horizon 60-120 $\mathrm{cm}$ (5 $200 \mathrm{t} / \mathrm{ha})$. Dans le profil D, la quantité de terre fine est plus élevée; elle représente $77 \%$ de la masse totale de l'horizon 0-30 $\mathrm{cm}$ ( $2400 \mathrm{t} / \mathrm{ha})$ et $74 \%$ de l'horizon $60-120$ $\mathrm{cm}$ (5 $200 \mathrm{t} / \mathrm{ha})$.

La granulométrie de la terre fine est relativement homogène dans les trois profils. Les pourcentages des fractions grossières augmentent avec la profondeur aux dépens des fractions fines. Cependant, la fraction d'argiles est importante en surface dans les profils C et D, et la fraction des sables grossiers est plus importante dans le profil D.

Les profils B et C présentent des densités apparentes de terre fine semblables de l'ordre de $0,74 \mathrm{~g} / \mathrm{cm}^{3}$ en surface, augmentant en profondeur pour atteindre la valeur de $1,1 \mathrm{~g} / \mathrm{cm}^{3}$. Le profil D se distingue des profils $B$ et $C$ par une densité de terre fine supérieure pour tous les horizons $\left(0,9 \mathrm{~g} / \mathrm{cm}^{3}\right.$ en surface et $1,2 \mathrm{~g} / \mathrm{cm}^{3}$ en profondeur).

\section{Répartition des nutriments dans le sol (tableau IV)}

\section{Stock total des éléments nutritifs}

Le stock total de potassium est le plus important, et cela dans chacune des trois classes granulométriques principales du sol (terre fine, gravillons et cailloux + blocs).

Le stock total de potassium est respectivement de 543, 481 et $656 \mathrm{t} / \mathrm{ha}$ dans les profils B, C et D. Dans ces derniers, le potassium est majoritairement localisé dans la terre fine (en moyenne $74 \%$ ). Ce stock est plus important dans les gravillons du profil $D(116 \mathrm{t} / \mathrm{ha}$ ) que dans les profils $\mathrm{B}$ et $\mathrm{C}$ (en moyenne $70 \mathrm{t} / \mathrm{ha}$ ). Alors que dans la classe des cailloux + blocs, ce stock est plus élevé dans le profil B (104 t/ha) que dans les profils C (51 t/ha) et D ( $19 \mathrm{t} / \mathrm{ha})$.

Le stock total de magnésium est respectivement de 118,87 et 105 t/ha dans les profils B, C et D. Dans les trois profils, la terre fine contient en moyenne $62 \%$ du stock tolal.

Le stock total de calcium est élevé dans le profil B ( $47 \mathrm{t} / \mathrm{ha}$ ) par rapport aux profils $\mathrm{C}$ et $\mathrm{D}$; il est également plus important dans le profil C (23 t/ha) que dans le profil D ( 10 t/ha). Le stock de calcium est localisé principalement dans les cailloux + blocs ( 30 $\mathrm{t} /$ ha) pour le profil $\mathrm{B}$, dans la terre fine $(9$ tha) pour le profil $C$ et dans les gravillons ( 5 t/ha) pour le profil $D$.

Le stock total de phosphore est respectivement de 6,7,5,2 et 6,5 t/ha dans les profils B, C et D. Il est majoritairement localisé dans la terre fine pour les profils $C$ et $D$ et dans les cailloux + blocs et la terre fine pour le profil $B$.

\section{Répartition des éléments nutritifs dans la terre fine (fig 2)}

Le potassium est toujours le nutriment le plus représenté dans les cinq fractions de la terre fine. Le stock total de potassium est essentiellement localisé dans la fraction des sables grossiers et des limons fins. La distribution du stock de potassium dans les fractions de la terre fine est relativement homogène au sein des trois profils. 
Tableau III. Répartition des classes granulométriques (t/ha à $110^{\circ} \mathrm{C}$ ) des sols de trois profils pédologiques (B, C, D) du massif des Aiguillettes.

\begin{tabular}{|c|c|c|c|c|c|c|c|c|c|c|c|c|c|}
\hline \multirow{2}{*}{\multicolumn{3}{|c|}{ Profondeur (en cm) TF }} & \multirow{2}{*}{$\frac{S O L}{G R}$} & \multirow[b]{2}{*}{$C B$} & \multirow[b]{2}{*}{$A R$} & \multirow[b]{2}{*}{$L F$} & \multirow{2}{*}{$\begin{array}{l}T F \\
L G\end{array}$} & \multirow[b]{2}{*}{$S F$} & \multirow[b]{2}{*}{$S G$} & \multirow[b]{2}{*}{$d(T F)$} & \multicolumn{3}{|c|}{$G R+C B$} \\
\hline & & & & & & & & & & & $G$ & $T S$ & $T A$ \\
\hline \multicolumn{14}{|l|}{ Profil B } \\
\hline \multirow{2}{*}{$0-10$} & t/ha & 556 & 129 & 108 & 108 & 154 & 41 & 66 & 187 & 0,62 & 109 & 23 & 104 \\
\hline & $\%$ & 70 & 16 & 14 & 14 & 19 & 5 & 8 & 24 & & 46 & 10 & 44 \\
\hline \multirow[t]{2}{*}{$10-30$} & t/ha & 1528 & 196 & 538 & 315 & 464 & 128 & 176 & 445 & 0,9 & 380 & 171 & 182 \\
\hline & $\%$ & 68 & 9 & 24 & 14 & 21 & 6 & 8 & 20 & & 52 & 23 & 25 \\
\hline \multirow[t]{2}{*}{$30-60$} & t/ha & 2559 & 445 & 842 & 469 & 762 & 215 & 343 & 771 & 1,03 & 562 & 297 & 427 \\
\hline & $\%$ & 67 & 12 & 22 & 12 & 20 & 6 & 9 & 20 & & 44 & 23 & 33 \\
\hline \multirow[t]{2}{*}{$60-120$} & $\mathrm{t} / \mathrm{ha}$ & 5270 & 1399 & 1835 & 572 & 1327 & 427 & 862 & 2080 & 1,13 & 2301 & 1336 & 1668 \\
\hline & $\%$ & 62 & 16 & 22 & 7 & 16 & 5 & 10 & 24 & & 7 & 41 & 52 \\
\hline \multicolumn{14}{|l|}{ Profil C } \\
\hline \multirow[t]{2}{*}{$0-10$} & t/ha & 555 & 102 & 93 & 118 & 166 & 51 & 62 & 158 & 0,6 & 118 & 9 & 68 \\
\hline & $\%$ & 74 & 14 & 12 & 16 & 22 & 7 & 8 & 21 & & 61 & 5 & 35 \\
\hline \multirow[t]{2}{*}{$10-30$} & $\mathrm{t} / \mathrm{ha}$ & 1430 & 224 & 310 & 351 & 434 & 114 & 173 & 359 & 0,87 & 354 & 28 & 151 \\
\hline & $\%$ & 73 & 11 & 16 & 18 & 22 & 6 & 9 & 18 & & 66 & 5 & 28 \\
\hline \multirow[t]{2}{*}{$30-60$} & t/ha & 2541 & 486 & 1077 & 563 & 672 & 192 & 343 & 771 & 1,07 & 1224 & 71 & 269 \\
\hline & $\%$ & 62 & 12 & 26 & 14 & 16 & 5 & 8 & 19 & & 78 & 5 & 17 \\
\hline \multirow[t]{2}{*}{$60-120$} & t/ha & 5090 & 1221 & 1312 & 603 & 1293 & 435 & 1035 & 1723 & 1,03 & 1403 & 106 & 1025 \\
\hline & $\%$ & 67 & 16 & 17 & 8 & 17 & 6 & 14 & 23 & & 55 & 4 & 40 \\
\hline \multicolumn{14}{|l|}{ Profil $D$} \\
\hline \multirow[t]{2}{*}{$0-30$} & t/ha & 2392 & 567 & 163 & 572 & 498 & 1.31 & 362 & 829 & 0,86 & 290 & 6 & 434 \\
\hline & $\%$ & 77 & 18 & 5 & 18 & 16 & 4 & 12 & 27 & & 40 & 1 & 59 \\
\hline \multirow[t]{2}{*}{$30-65$} & tha & 4282 & 982 & 330 & 918 & 886 & 238 & 717 & 1524 & 1,38 & 611 & 13 & 689 \\
\hline & $\%$ & 77 & 18 & 6 & 16 & 16 & 4 & 13 & 27 & & 47 & 1 & 52 \\
\hline \multirow[t]{2}{*}{$65-115$} & $\mathrm{t} / \mathrm{ha}$ & 5200 & 1577 & 277 & 898 & 1021 & 290 & 977 & 2014 & 1,2 & 363 & 48 & 1443 \\
\hline & $\%$ & 74 & 22 & 4 & 13 & 14 & 4 & 14 & 29 & & 20 & 3 & 78 \\
\hline
\end{tabular}

Terre fine (TF); gravillons (GR : 2-20 mm); cailloux + blocs $(\mathrm{CB}:>20 \mathrm{~mm}) ; \operatorname{argiles}(\mathrm{AR}:<2 \mu \mathrm{m})$;

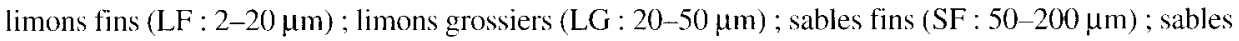
grossiers (SG : 200-2000 $\mu \mathrm{m}$ ) ; d(TF) : densité de la terre fine ; Grès (G) ; tuf sain (TS) ; tuf altéré (TA).

Dans les trois profils, le stock du magnésium est principalement localisé dans les fractions d'argiles et de limons fins.

Le stock de calcium se trouve en grande partie dans la fraction des limons fins et des sables grossiers. Il est faible dans la frac- tion d'argiles, particulièrement dans l'horizon de surface des profils B et C $(0,03$ tha).

Le stock de phosphore est important dans les fractions d'argiles, de limons fins et de sables grossiers. Il représente en moyenne 3 t/ha dans les profils B et C et 1,8 t/ha dans le profil D. 


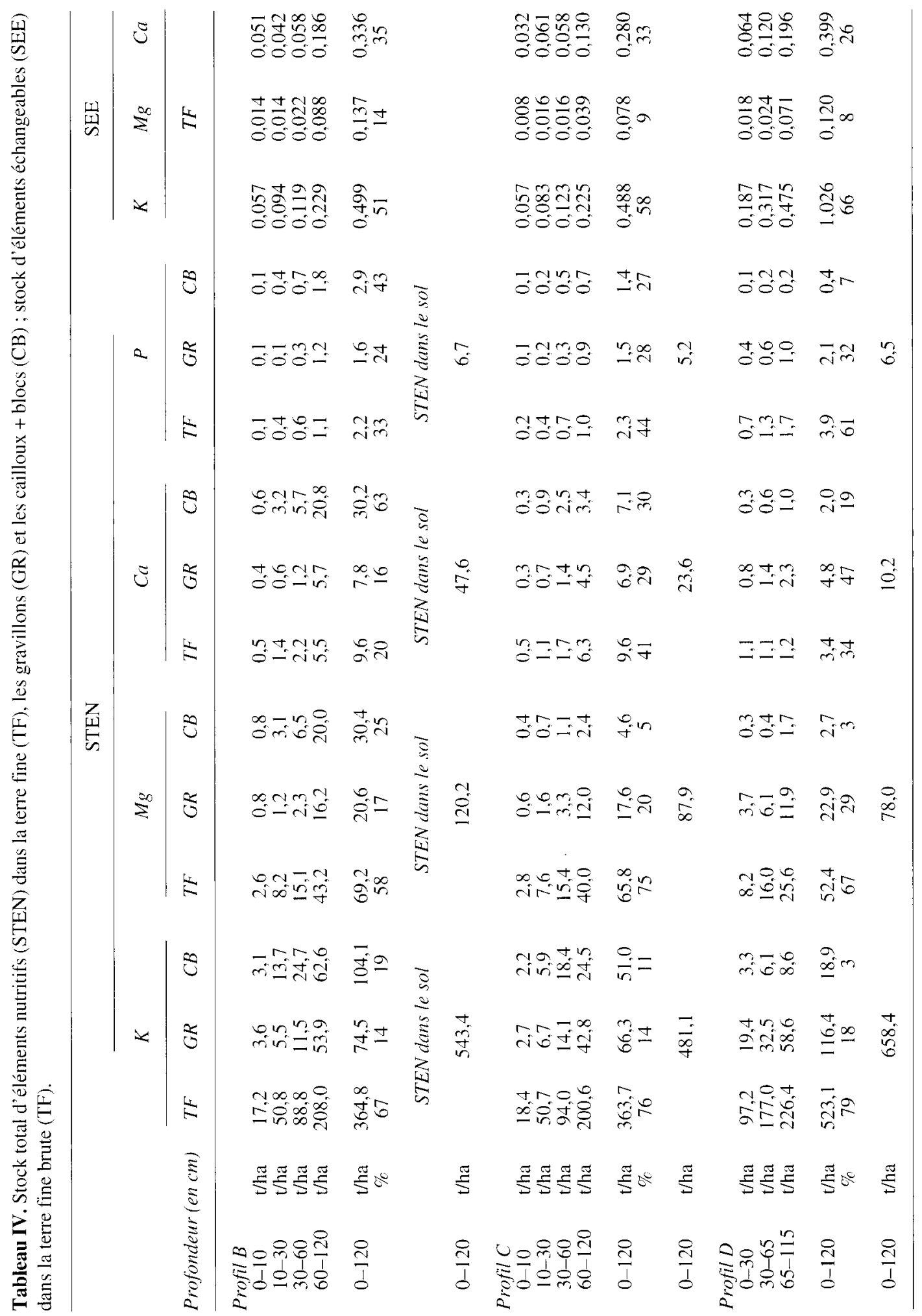



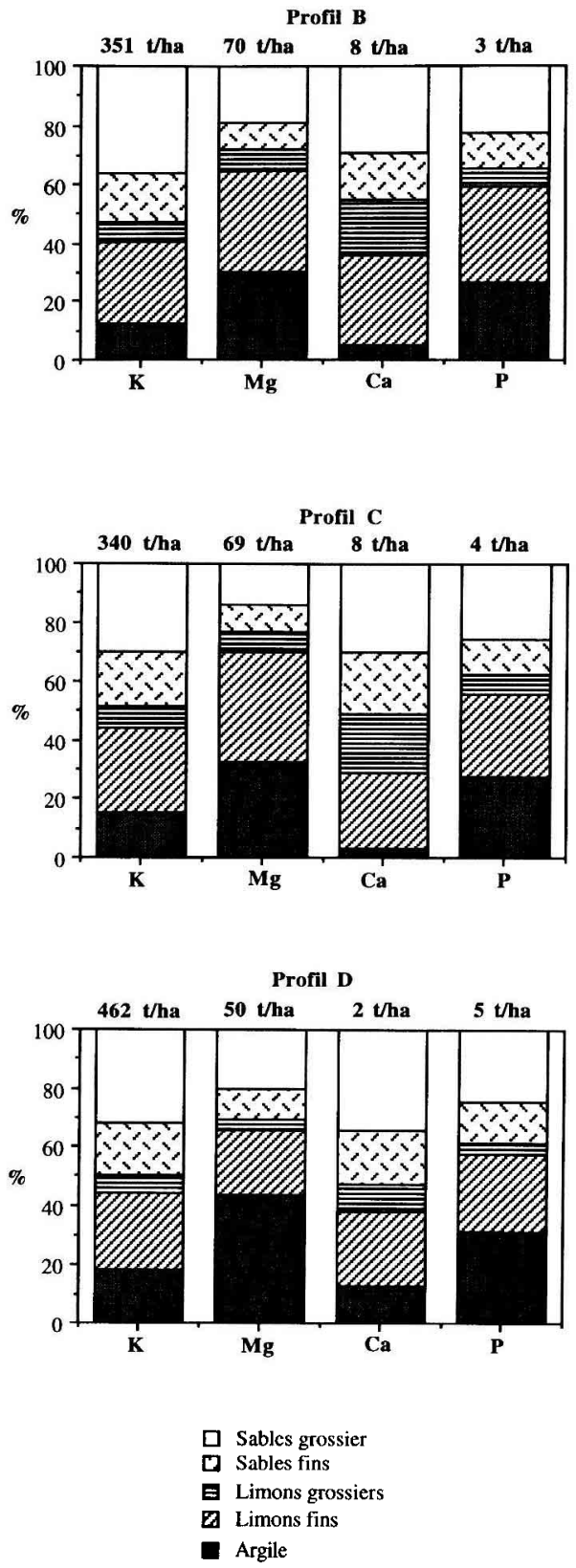

Fig 2. Répartition du stock d'éléments nutritifs ( $\mathrm{K}, \mathrm{Mg}, \mathrm{Ca}$ et $\mathrm{P}$ ) dans les fractions de la terre fine de l'horizon $0-120 \mathrm{~cm}$.

\section{Stock d'éléments échangeables dans la terre fine}

La capacité d'échange cationique (T) du sol a une valeur comprise entre 4 et $6 \mathrm{cmol}_{\mathrm{c}} / \mathrm{kg}$ de terre fine, et exceptionnellement, de 10 $\mathrm{cmol} / \mathrm{kg}$ dans l'horizon $0-10 \mathrm{~cm}$ du profil $\mathrm{B}$ (tableau II). Le taux de saturation (S/T) augmente en profondeur et peut atteindre $20 \%$ dans les horizons les plus profonds. Il est inférieur à $15 \%$ jusqu'à $1 \mathrm{~m}$ de profondeur, ce qui est caractéristique des sols désaturés (tableau II).

Le stock d'éléments nutritifs représenté par les ions échangeables de la terre fine est composé principalement par le potassium, le calcium et, à un moindre degré, par le magnésium pour les trois profils.

Le stock de potassium est deux fois plus important dans le profil D ( $1026 \mathrm{~kg} / \mathrm{ha}$ ) que dans les profils $\mathrm{B}$ et $\mathrm{C}$ (tableau IV).

Le stock de calcium échangcable est aussi plus élevé dans le profil D $(400 \mathrm{~kg} / \mathrm{ha})$ que dans les profils B et C. Il est également important dans le profil B (337 kg/ha) par rapport au profil C (280 kg/ha ; tableau IV).

Le stock de magnésium échangeable est supérieur dans le profil B (137 kg/ha) par rapport aux profils $C$ et $D$. Il est également plus important dans le profil D $(120 \mathrm{~kg} / \mathrm{ha})$ que dans le profil C ( $78 \mathrm{~kg} / \mathrm{ha}$; tableau IV).

\section{Répartition des éléments nutritifs dans la fraction supérieure à $2 \mathrm{~mm}$ en fonction des roches (fig 3 )}

Le potassium représente toujours le stock total le plus important dans les deux roches (tuf et grès) présentes dans les trois profils. Le stock total de potassium dans la fraction supérieure à $2 \mathrm{~mm}$ est respectivement de 178,117 et 135 t/ha dans les profils $\mathrm{B}, \mathrm{C}$ et D. Il se trouve en grande partie dans le tuf sain et dans le tuf altéré pour le profil B, dans le tuf altéré et dans le grès pour le profil C et dans le tuf altéré pour le profil D. 
Profil B
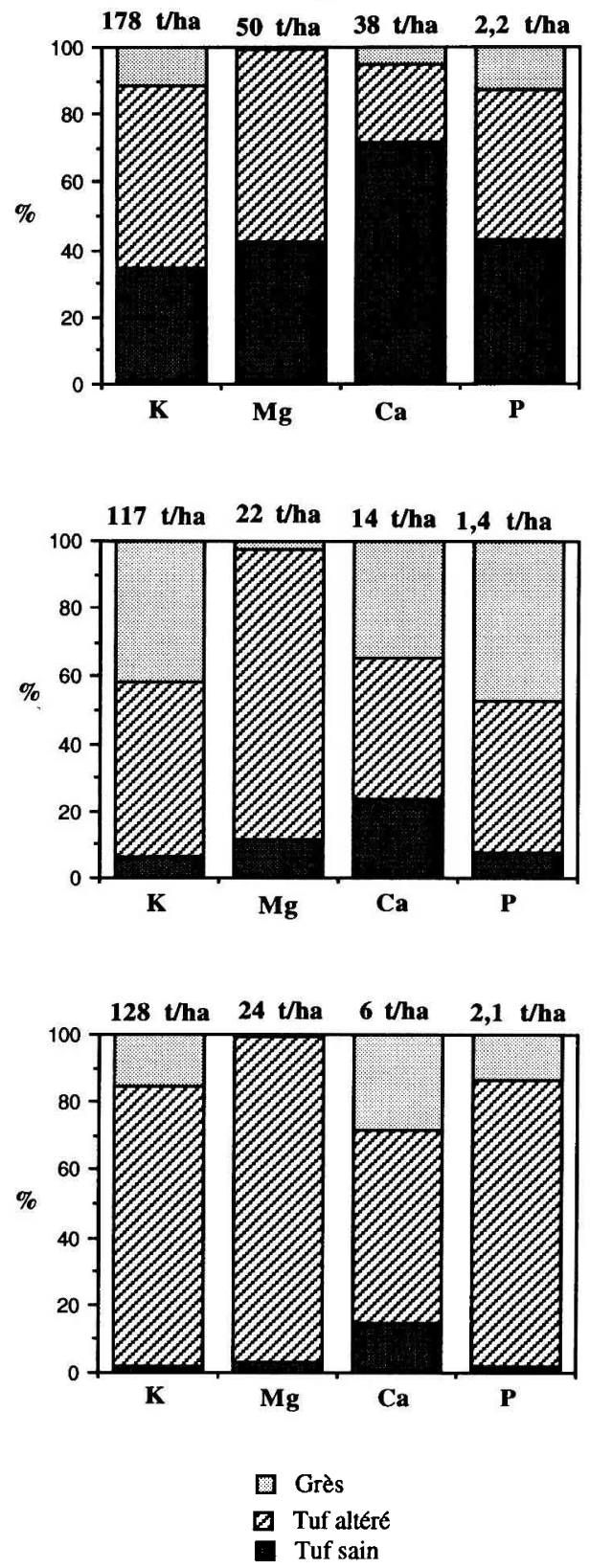

Fig 3. Répartition du stock d'éléments nutritifs $(\mathrm{K}, \mathrm{Mg}, \mathrm{Ca}$ et $\mathrm{P})$ dans le grès, le tuf altéré et le tuf sain de la fraction supérieure à $2 \mathrm{~mm}$ de l'horizon $0-120 \mathrm{~cm}$.
Le stock total de calcium est plus élevé dans le profil $\mathrm{B}$ ( $38 \mathrm{t} / \mathrm{ha}$ ) que dans les profils $C$ et $D$ (en moyenne $10 \mathrm{t} / \mathrm{ha}$ ). Il est localisé essentiellement dans le tuf sain pour le profil B et dans le tuf altéré pour le profil D. Cependant, il est réparti d'une manière relativement homogène entre le tuf sain, le tuf altéré et le grès dans le profil $\mathrm{C}$.

Le stock total de magnésium est respectivement de 51,22 et 25 t/ha dans les profils $\mathrm{B}, \mathrm{C}$ et $\mathrm{D}$. Il se trouve presque en totalité dans le tuf sain et dans le tuf altéré pour le profil $\mathrm{B}$, dans le tuf altéré pour les profils C et D.

Le stock de phosphore est plus important dans le profil $\mathrm{B}(4,5 \mathrm{t} / \mathrm{ha})$ que dans les profils $C$ et $D$ (cn moyenne $2,7 \mathrm{t} / \mathrm{ha}$ ). Il est concentré majoritairement dans le tuf sain et dans le tuf altéré pour le profil $\mathrm{B}$, dans le tuf altéré et dans le grès pour le profil $\mathrm{C}$ et dans le tuf altéré pour le profil D.

\section{DISCUSSION}

La démarche analytique présentée précédemment, relativement lourde, a été effectuée sur un seul profil par placette. Cependant, la comparaison avec les analyses granulométriques, de chimie totale, de capacité d'échange cationique et du taux de saturation de trois ou sept profils par peuplement (Marques, 1996) montre que les profils B, C et D choisis sont représentatifs des placettes étudiées.

\section{Granulométrie}

La granulométrie et la quantité de cailloux, de grès et de tuf altéré des sols des trois profils étudiés permettent de déterminer l'intensité de la dégradation physique de la roche mère et du taux de colluvionnement.

La transformation de la terre fine d'un sol dépendant de l'intensité relative de la fragmentation et de la dissolution (Legros, 
1982), deux indices traduisant ces mécanismes ont été définis en tenant compte de l'ensemble des fractions des sols étudiés.

Un indice de microdivision (IM) a été établi arbitrairement à partir du rapport des taux pondérés des différentes fractions de la terre fine $(<2 \mathrm{~mm})$ et des classes granulométriques de la fraction grossière ( $>2$ $\mathrm{mm}) \mathrm{du}$ sol :

$$
\mathrm{IM}=\frac{(\mathrm{AR} * 1000)+(\mathrm{LF} * 100)+\mathrm{LG}}{\mathrm{SF}+\mathrm{SG}+(\mathrm{GR} * 100)+(\mathrm{CB} * 1000)}
$$

Dans le profil D, cet indice de microdivison est nettement plus élevé $(>200)$ que dans les deux autres profils, car la quantité d'argiles y est importante et la quantité de cailloux + blocs y est faible (fig 4). Cet indice diminue avec la profondeur. Dans les horizons de surface, il est légèrement plus élevé dans le profil C que dans le profil B.
De plus, un indice d'altération supergène (IA) est défini par le rapport entre la quantité du tuf altéré (TA) et du tuf sain (TS). Les deux indices sont corrélés positivement et suivent la même évolution en fonction de la profondeur (fig 4).

Le tuf hydrothermalisé (profil D) présente une dégradation physique intense qui se traduit par un fort indice de microdivision. Le pourcentage de tuf hydrothermalisé non affecté par l'altération supergène est très faible $(<3 \%)$, d'où le fort indice d'altération. Cette intense altération supergène peut s'expliquer par les propriétés physiques du tuf hydrothermalisé (perméabilité importante). De plus, la présence des micas blancs hydrothermaux de petite taille $(<5$ $\mu \mathrm{m})$ au sein des phénocristaux et du verre du tuf hydrothermalisé contribue à la forte teneur en argile dans le profil D. À l'inverse,

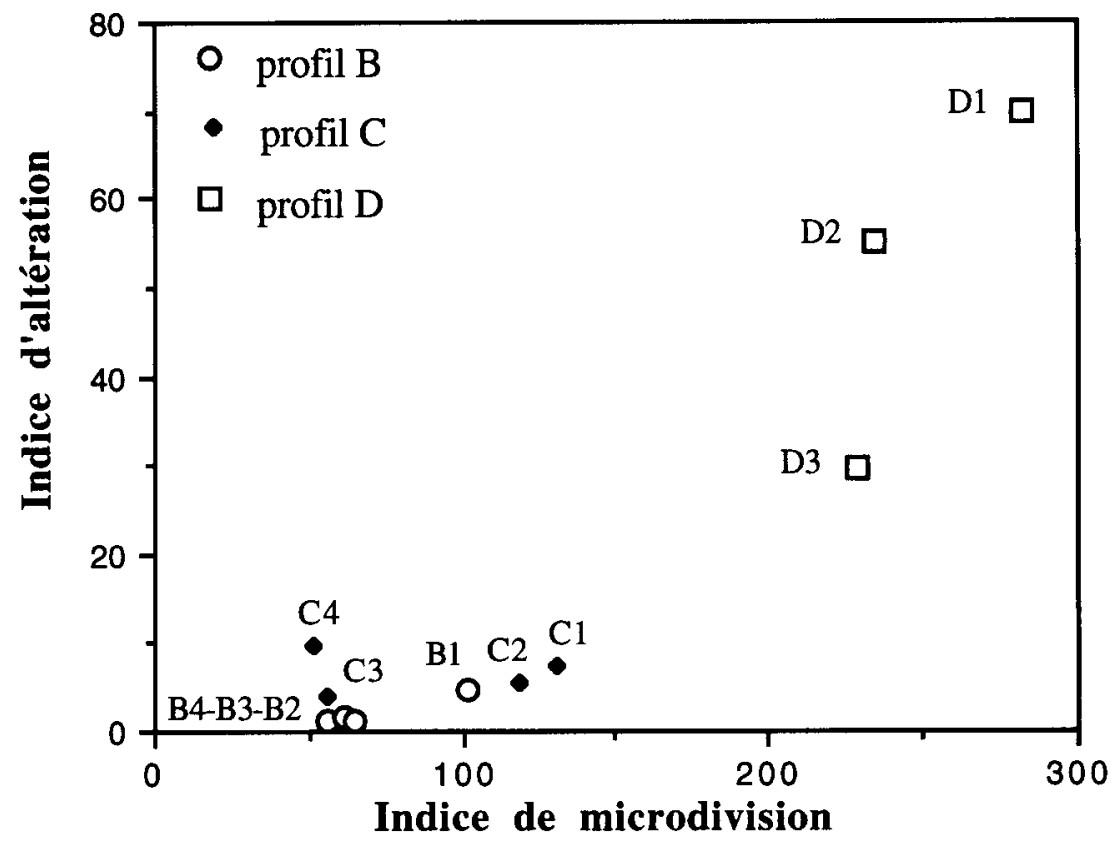

Fig 4. Relation entre l'indice de microdivision (IM) et l'indice d'altération (IA) en fonction de la profondeur ( 1 : horizon de surface ; 4 : horizon profond). 
dans le profil $\mathrm{B}$ développé à partir du tuf non hydrothermalisé, les indices de microdivision et d'altération sont faibles. Cela peut s'expliquer par le mécanisme d'altération supergène du tuf qui, dans les premiers stades, résulte principalement d'une altération chimique du fait des propriétés physiques de cette roche massive peu apte à la microdivision. Son altération se traduit par le développement d'une pellicule d'épaisseur inférieure à $\mathrm{I} \mathrm{cm}$ à la périphérie des blocs de tuf sain. La desquamation de cette pellicule donne ensuite naissance aux fractions grossières de la terre fine (Ezzaïm et al, soumis pour publication).

La figure 4 montre que les indices de microdivision et d'altération dans les horizons de surface du profil $\mathrm{C}$ sont intermédiaires entre ceux du profil $B$ et ceux du profil $D$. Cependant, la roche mère en place est la même dans les profils $B$ et $C$ (tuf non hydrothermalisé). Le taux d'argiles élevé dans le profil C peut s'expliquer par un colluvionnement de terre fine issu du toit du tuf hydrothermalisé.

La forte quantité de grès dans le profil $\mathrm{C}$ témoigne vraisemblablement d'un colluvionnement ( 50 à $80 \%$ des cailloux + blocs sont du grès). Dans ce profil, le pourcentage de graviers est à peu près stable tout au long du profil, alors que le taux de cailloux augmente fortement dans l'horizon $50-100 \mathrm{~cm}$ (tableau IV). Cette augmentation est due à la présence d'un lit de blocs de grès. Le colluvionnement du grès issu de la montagne des Aiguillettes ne semble pas pouvoir expliquer seul le phénomène. Le profil C est situé dans une parcelle qui a été cultivée jusqu'en 1930 et ensuite boisée par l'actuel peuplement de Douglas. Indépendamment de l'altitude et de la situation topographique du profil $\mathrm{C}$ et du colluvionnement, la distribution des classes granulométriques de la fraction grossière du sol ( $>2 \mathrm{~mm}$ ) a pu être perturbée par l'intervention humaine (Koener, communication personnelle). Le colluvionnement des profils (B, D) semble uniquement d'origine géomorphologique et n'affecte que les horizons de surface $(0-60 \mathrm{~cm})$.

\section{Relation entre le stock des éléments nutritifs et l'évolution de la fertilité dans une plantation de douglas}

L'immobilisation brute des éléments nutritifs dans un peuplement de douglas de 60 ans correspond à des quantités importantes de calcium (391 kg/ha) et de potassium (207 $\mathrm{kg} / \mathrm{ha}$ ) et à des quantités plus modérées de magnésium ( $42 \mathrm{~kg} / \mathrm{ha}$ ) et de phosphore (41 $\mathrm{kg} / \mathrm{ha}$ ) (Ranger et al, 1995b).

Les apports atmosphériques totaux cumulés pour ce peuplement sont trop faibles pour pouvoir compenser en totalité les pertes cumulées d'éléments nutritifs par drainage à $-120 \mathrm{~cm}$ pendant 60 ans (fig 5 ; Marques, 1996). Supposant que le complexe adsorbant et le compartiment de matière organique (horizons holorganiques $\mathrm{L}+\mathrm{H}$ ) sont stables, le flux d'altération doit obligatoirement compenser la totalité du déficit des bilans entrées-sorties pour maintenir l'écosystème dans son état actuel de stabilité. $\mathrm{Si}$ le compartiment de matière organique est instable, le déficit du bilan va être équilibré à la fois par la dégradation de la matière organique, par le flux d'altération et éventuellement par la désaturation du complexe adsorbant du sol.

Le stock de calcium apparaît le plus limitant pour la nutrition du douglas (figs 2 et 5 ). Dans le profil B, le stock de calcium de la fraction d'argiles dans l'horizon $0-120 \mathrm{~cm}$ correspond à une révolution forestière $(0,4$ t/ha). Il est vraisemblable que le flux de calcium issu des minéraux de cette fraction argileuse (F4) ne comblera pas tout seul les exigences de l'essence en cet élément durant une révolution forestière. Les besoins du peuplement de Douglas nécessiteront donc un flux de calcium issu des fractions gros- 


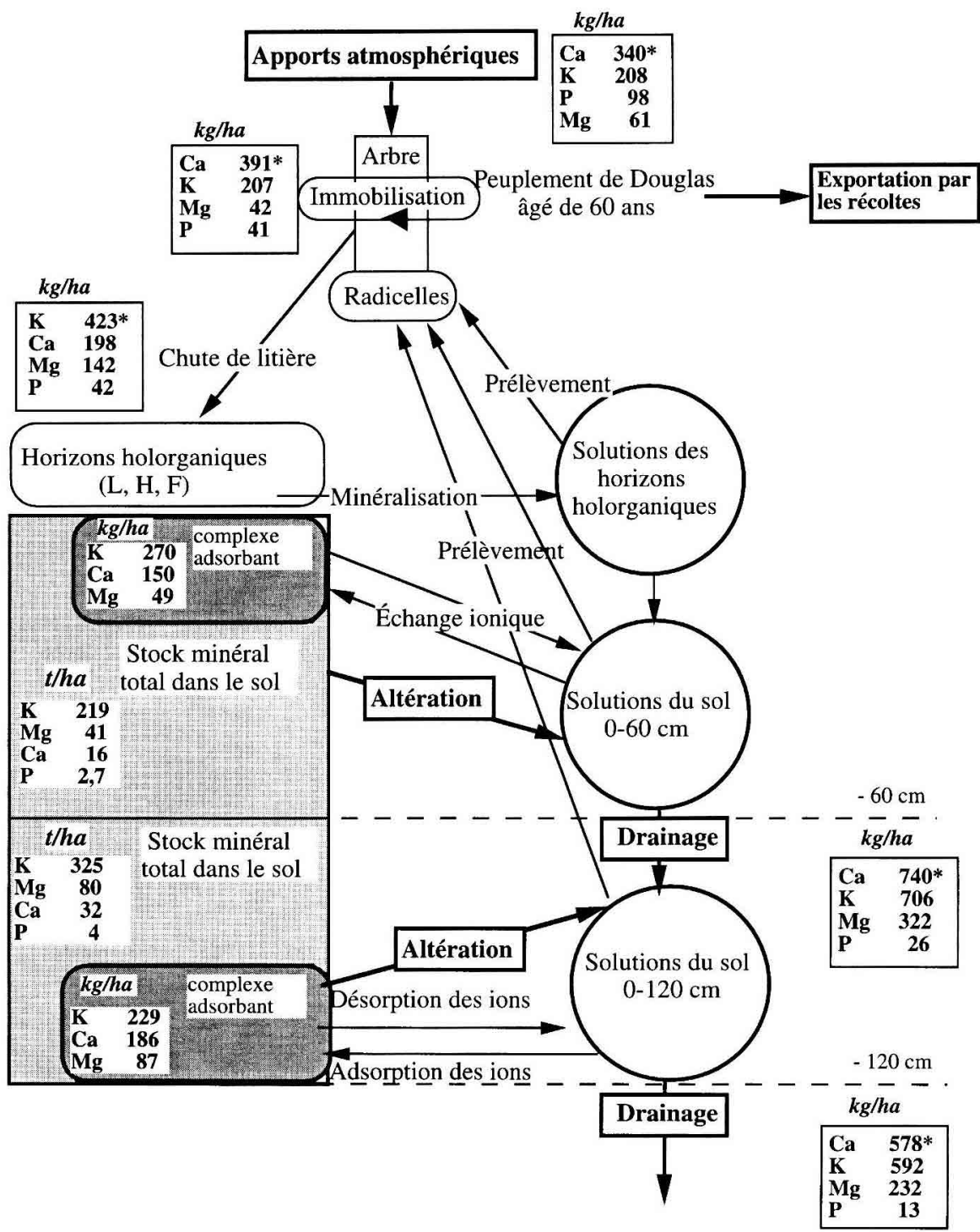

Fig 5. Stock de nutriments dans le sol et entrées-sorties cumulées pour un peuplement de douglas de 60 ans ; * données fournies par Marques, 1996 (modèle adapté de Ranger et Bonneau, 1984).

sières $(\mathrm{F} 1, \mathrm{~F} 2, \mathrm{~F} 3)$ qui représente la fertilité à moyen et à long terme (fig 6).

Les minéraux secondaires ne contenant pas de calcium, le flux de cet élément dépend de la vitesse de dissolution des minéraux primaires calciques (plagioclase, épidote et apatite ; Ezzaïm et al, soumis pour publication). Les surfaces spécifiques des 


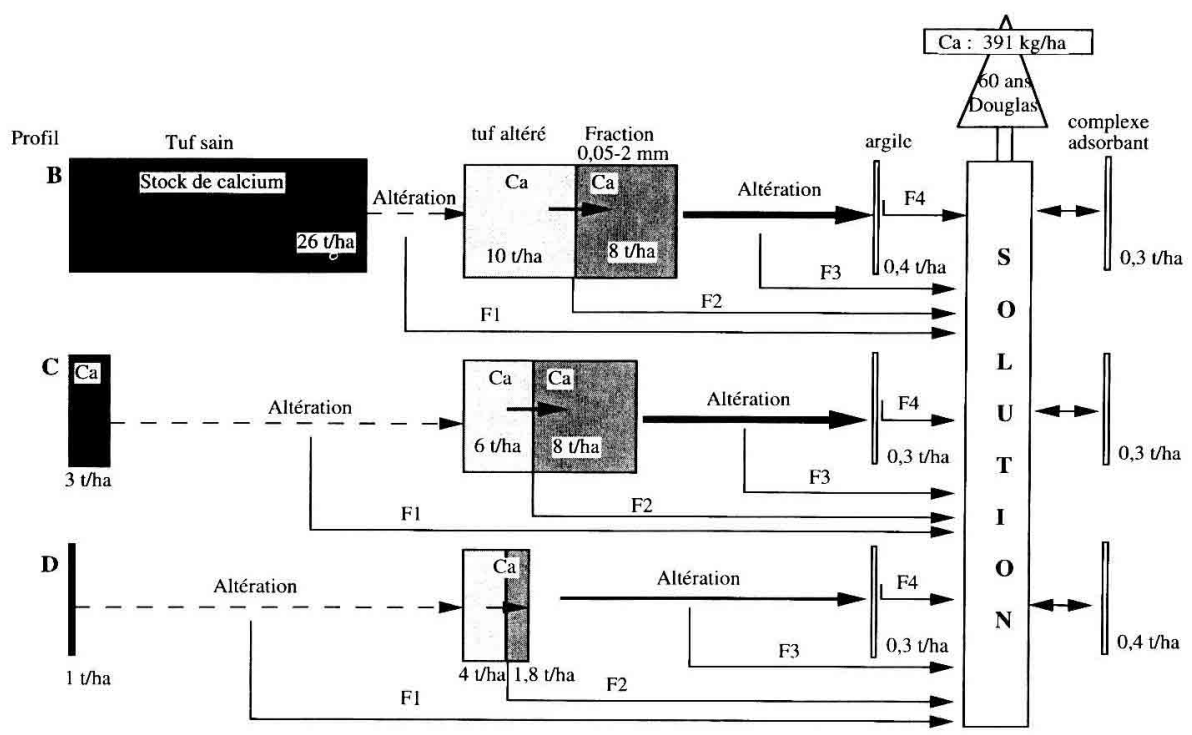

Fig 6. Estimation du flux de calcium théorique (F1, F2, F3, F4) en fonction de l'importance et de la nature des stocks (en tha) dans les différentes classes granulométriques du sol et sur le complexe adsorbant $(0-120 \mathrm{~cm})$ des trois profils $(B, C, D)$.

minéraux primaires calciques étant bien plus élevées dans le tuf altéré + terre fine que dans le tuf sain, la somme des flux F2 et F3 de calcium est supérieure au flux F1. Le stock total de calcium dans le profil $\mathrm{C}$ est intermédiaire entre les profils B et D. Il est très faible dans le profil $\mathrm{D}$. Il est probable que les différents flux (F1, F2, F3, F4) de calcium dans ce profil soient insuffisants pour maintenir la réserve temporaire de calcium sur le complexe adsorbant (fertilité à court terme) et pour compenser le prélèvement par la végétation à long terme (fig 6).

Le stock de calcium du tuf sain est très important dans le profil B. Le flux de calcium Fl est proportionnel à la vitesse de formation de la pellicule d'altération autour des cailloux sains. La vitesse de desquamation de cette pellicule contrôle la quantité de la fraction inférieure à $1 \mathrm{~cm}$ et, par conséquent, le flux de calcium issu de cette fraction.
La stabilité de cet écosystème doit se traduire par l'équilibre entre les entrées et les sorties. Elle dépend donc en grande partie de l'importance du flux d'altération issu des minéraux des fractions grossières de la terre fine $(>2 \mu \mathrm{m})$, des gravillons et des cailloux + blocs (fertilité à long terme).

\section{CONCLUSION}

L'appréciation de la fertilité à court terme d'un sol est souvent limitée à la quantification des éléments nutritifs échangeables, alors que l'évaluation de la fertilité à moyen et à long terme est plus rarement effectuée. Selon la définition des classes de fertilité chimique à court terme (Bonneau, 1995), pour les trois profils étudiés, le stock des nutriments échangeables ( $\mathrm{Ca}, \mathrm{Mg}$ et $\mathrm{K}$ ) correspond à la classe la plus faiblement pourvue. 
Cette étude montre que la fertilité à moyen et à long terme est très différente dans ces profils, bien que leurs stocks en nutriments échangeables soient comparables. La fertilité à moyen et à long terme correspond aux stocks d'éléments nutritifs dans le tuf sain, dans la pellicule d'altération et dans les fractions granulométriques issues de cette pellicule $(<1 \mathrm{~cm})$.

Le stock minéral de potassium et, dans une moindre mesure, de magnésium dans le sol est important par rapport aux exigences nutritives du douglas. Le calcium apparaît comme l'élément nutritif le plus limitant dans cet écosystème, malgré sa teneur élevée dans la roche mère.

Étant donné l'absence de minéraux secondaires calciques, le flux de calcium dépend uniquement de la dissolution des minéraux primaires calciques du sol.

Quel que soit le profil, le stock de calcium dans la fraction argileuse est faible et insuffisant pour maintenir à long terme la réserve de calcium sur le complexe d'échange, seuls les flux de calcium issu des fractions grossières du sol ( $>2 \mu \mathrm{m})$ sont capables de compenser l'immobilisation dans la biomasse du douglas. Les stocks de calcium dans les fractions grossières sont très différents dans les trois profils. Dans le profil B, le stock de calcium dans le tuf sain est 22 fois supérieur à celui du profil $\mathrm{D}$; de même, ce stock dans le tuf altéré est deux fois supérieur à celui du profil D. Le stock de calcium dans les fractions grossières pour le profil C est intermédiaire entre les deux autres profils. La compensation de l'immobilisation des éléments nutritifs dans la biomasse par le flux d'altération est improbable à long terme dans le profil D. L'hydrothermalisme intense du tuf au niveau de ce profil conduit à un appauvrissement en calcium et à une forte altération physicochimique de la roche. Le colluvionnement du profil $\mathrm{C}$ par ce tuf fortement hydrothermalisé entraîne également un appauvrissement en calcium par rapport au profil $\mathrm{B}$.
L'ensemble de ces données, couplé aux analyses minéralogiques, va permettre de quantifier les minéraux dans le sol et d'effectuer une mesure du flux de cations issu de leur altération (F1, F2, F3, F4; fig 6) à l'aide du modèle géochimique Profile (Sverdrup et Warfvinge, 1988). Cela fera l'objet d'un prochain article.

\section{REMERCIEMENTS}

Nous tenons à remercier les collègues de l'équipe Cycles biogéochimiques pour leur aide dans les séparations granulométriques du sol.

\section{RÉFÉRENCES}

AFES (1992) Référentiel pédologique. Principaux sols d'Europe. Inra, Paris

Bertaux J, Rubiello MF (1981) Cartographie de la feuille géologique de Roanne au 1/50 000, pétrologie, géochimie et pétrologie structurale du magmatisme du Viséen supérieur. Essai de synthèse régionale. Thèse univ, Nancy I

Bonneau M (1995) Fertilisation des forêts dans les pays tempérés. Engref, Nancy

Espiau P, Peyronel A (1976) L'acidité d'échange des sols ácides, le taux d'acidité d'échange et sa signification pédologique sous climat tempéré. Ann Agron 31, 363-383

Legros JP (1982) L'évolution granulométrique au cours de la pédogenèse. Approche par simulation sur ordinateur. Application aux sols acides sur matériaux cristallins en zone tempérée. Thèse, univ Monpellier

Marques $R$ (1996) Dynamique du fonctionnement minéral d'une plantation de douglas (Pseudotsuga menziessii MIRB Franco) dans les monts du Beaujolais (France). Thèse, Engref, Nancy

Mehra OP, Jackson ML (1960) Iron oxide removal from soils and clays by a dithionite-citrate system buffered with sodium bicarbonate. Clays Clay Miner 7, 317-327

Morel B (1976) Les formations éruptives dévonodinantiennes de la bordure ouest du bassin d'Autin (Saône-et-Loire, France). Thèsce, univ Claude-Bernard-Lyon-I

Ranger J, Bonneau M (1984) Effets prévisibles de l'intensification de la production et des récoltes sur la fertilité des sols de forêts. Le cycle biologique en forêt. Rev For Fr 2, 93-11

Ranger J, Colin-Belgrand M, Nys C (1995a) Le cycle biogéochimique des éléments majeurs dans les éco- 
systèmes forestiers, importance dans le fonctionnement des sols. Étude et Gestion des Sols 2, 119 134

Ranger J, Marques R, Colin-Belgrand M, Flammang N (1995b) The dynamics of biomass and nutrient accumulation in a Douglas fir (Pseudotsuga menziessii Franco) stand studied using a chronosequence approch. For Ecol Manage 72, 167-183

Robert M, Tessier D (1974) Méthode de préparation des argiles des sols pour l'étude minéralogique. Ann Agron 22, 43-93
Sider H, Leistel JM (1986) Réflexion sur l'évolution géodynamique de la chaîne hercynienne au paléozoïque dans le nord-est du Massif central français. Bull Soc Géol Fr 8, 637-644

Stanley A, Barber A (1984) Soil Nutrient Bioavailability. A Mechanistic Approach. Wiley, New York

Sverdrup H, Warfvinge P (1988) Weathering of primary silicate minerals in the natural soil environment in relation to a chemical weathering model. Water Air Soil Pollut 38, 387-408

Tamura $T$ (1958) Identification of clay minerals from acid soils. $J$ Soil Sci $9,141-147$ 